DISCUSSION

\title{
Revision of effective stress method of slices
}

\author{
G. J. W. KING (1989). Géotechnique 39, No. 3, 497-502
}

S. K. Sarma, Imperial College

NOTATION

$W_{1}$ weight of soil column above phreatic line

$W_{2}$ weight of soil column below phreatic line $\left(=W_{2}^{\prime}+W_{\mathrm{w}}\right)$

$W_{\mathrm{w}}$ weight of water column below phreatic line

$W_{2}^{\prime} \quad$ submerged weight of soil column below phreatic line

$X$ inter-slice shear force

$\Delta X$ increment of inter-slice shear force across slice

$E$ inter-slice thrust in terms of total stresses $\left(=E^{\prime}+P_{\mathrm{w}}\right)$

$E^{\prime}$ inter-slice thrust in terms of effective stresses

$P_{\mathrm{w}}$ force due to pore water pressure on side of slice

$\Delta E$ increment of total inter-slice thrust across slice $\left(=\Delta E^{\prime}+\Delta P_{\mathrm{w}}\right)$

$\Delta E^{\prime}$ increment of effective inter-slice thrust across slice

$\Delta P_{w}$ increment of pore water force on side across slice

$N$ Normal force on the base of the slice in terms of total stresses

$N^{\prime}$ normal force on the base of the slice in terms of effective stresses

$U$ force due to pore water pressure on base of slice

$u$ average pore water pressure on base of slice $(=U /(b \sec \alpha))$

$T$ shear force on base of slice

$F_{\mathrm{s}} \quad$ secpage force on slice

$F_{\mathrm{s}} \sin \beta \quad W_{\mathrm{w}}-U \cos \alpha$

$F_{\mathrm{s}} \cos \beta \quad \Delta P_{\mathrm{w}}+U \sin \alpha$

The Author has correctly pointed out the need to take into account the seepage forces in the stability analysis of slopes by the method of slices. However, he gives the impression that all other methods of slices are wrong on this account. The point to be noted is that all rigorous methods are implicitly correct when inter-slice shear and thrust are taken into account. It is immaterial whether the thrusts are considered as total or effective together with the force due to pore water pressure. It can be shown that when the force due to pore water pressures is correctly accounted for on the base as well as in the vertical sides of the slice, then the equilibrium of the body and seepage forces as well as the normal and shear forces is maintained, both in terms of effective stresses and total stresses. The error, if any, therefore appears when the inter-slice water forces are not computed properly, for example when the piezometric head on the slip surface is confused with the phreatic line. Sarma (1973) has shown clearly that the water force on the vertical faces must be calculated from the piezometric head, taking into account the variation of the head at different levels, e.g. from a flow net. Fig. 7 explains the equivalence of the Author's proposed method and existing rigorous methods. It is clear that there is no difference between the two approaches regarding the vertical component of the seepage force. If the force due to pore water pressure is computed properly on the vertical sides of the slice, then there is no difference in the horizontal component either. Moreover, in the rigorous solutions of the slope stability methods, such as of Sarma (1973), Morgenstern \& Price (1965), Janbu (1957) or Bishop (1955) etc., the difference, if any, in the horizontal component of the seepage force does not affect the factor of safety; it affects only the local inter-slice factors of safety.

The problem that the Author addresses is therefore one in the simplified methods such as those proposed by Fellenius (1927, 1936), Bishop (1955, simplified), Janbu (1957, simplified), etc., where simplifying assumptions are made to facilitate the solution. One can therefore argue that the treatment of the seepage forces is part of the same assumption, albeit unknowingly. In fact, the simplified methods of Bishop and Janbu will both give different solutions which are independent of the horizontal component of the seepage forces. However, these methods do not satisfy horizontal equilibrium.

Regarding partially submerged slopes, the technique of solution proposed by Bishop (1955) is perfectly correct and applicable to both circular and non-circular slip surfaces, provided pore pressures are taken care of correctly. I am not aware of a published proof of this method other than the one given by Bishop for circular arc slip 

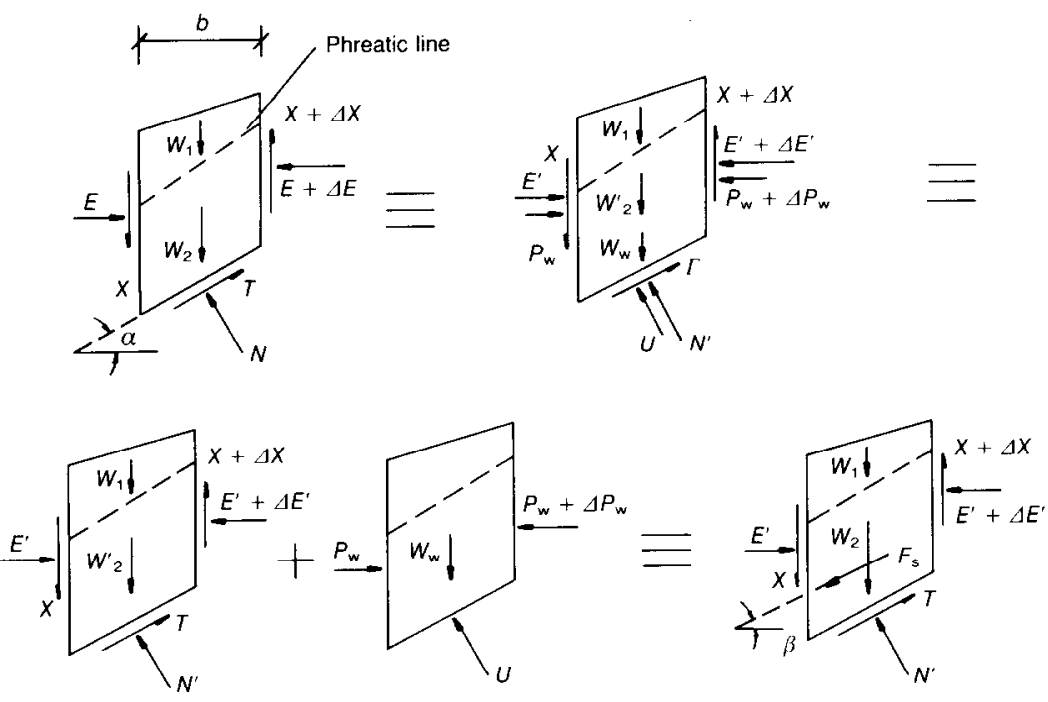

Fig. 7. Equivalence of King's proposed method and existing rigorous methods (see notation)

surfaces. A formal proof of this method is given here. It is assumed that we are applying this technique in a rigorous solution, as Sarma (1973).

Let us assume that the problem, as shown in Fig. 8(a), is solved correctly and all the forces in terms of effective stresses and pore water pressures are obtained. The mass above the slip surface is now in equilibrium with the applied body, seepage and external forces and the resultant stresses. This equilibrium state can be shown easily in the vector statement

$$
\begin{aligned}
\bar{W}_{\mathrm{s} 1}+\bar{W}_{\mathrm{s} 2}+\bar{W}_{\mathrm{we}}+\bar{P}_{\text {we }} & +\Sigma \bar{N}^{\prime} \\
& +\Sigma \bar{U}+\Sigma \bar{T}=0
\end{aligned}
$$

In this state of equilibrium, the internal effective thrusts, the inter-slice pore water forces and the inter-slice shear forces do not play any part, other than contributing to the normal effective and shear stresses on the slip surface. This equilibrium is not disturbed if we write the equation as

$$
\begin{aligned}
\bar{W}_{\mathrm{s} 1}+\bar{W}_{\mathrm{s} 2}^{\prime}+\bar{W}_{\mathrm{w} 2}+\bar{W}_{\mathrm{we}} & +\bar{P}_{\mathrm{we}}+\Sigma \bar{N}^{\prime} \\
& +\Sigma \bar{U}+\Sigma \bar{T}=0
\end{aligned}
$$

In this equation $W_{\mathrm{s} 2}=W_{\mathrm{s} 2}{ }^{\prime}+W_{\mathrm{w} 2}$, where $W_{\mathrm{s} 2}^{\prime}$ is the submerged weight of the soil below the level of the external water, which does not mean that the soil is actually submerged except in the case of the hydrostatic water. $W_{w 2}$ is the weight of water for the same volume as that for $W_{\mathrm{s} 2}$.

The resultant of the external water (both vertical load $W_{\text {we }}$ and the horizontal force $P_{\text {we }}$ ) and the water load $\left(+W_{w 2}\right)$ is equal to a pressure $\left(\gamma_{w} h\right)$ on that part of the slip surface which is below the level of the external water where $h$ is the height of the external water level above the slip surface and $\gamma_{w} h$ acts normal to the slip surface and in the opposite direction to the actual pore water pressure $\gamma_{w} h_{1}$, as shown in Fig. 8(b). The term $\gamma_{w} h$ is a fictitious pressure and does not actually exist. The normal effective forces and the shear forces are unaffected. Therefore, we obtain

$$
\bar{W}_{\mathrm{s} 1}+\bar{W}_{\mathrm{s} 2}{ }^{\prime}+\Sigma \bar{N}^{\prime}+\Sigma \bar{U}_{1}+\Sigma \bar{T}=0
$$

where $U_{1}=\gamma_{\mathrm{w}}\left(h_{1}-h\right) b \sec \alpha$ and $b$ is the width and $\alpha$ is the inclination of the base of the slice as shown in Fig. 7. Therefore, the equilibrium of the soil mass is unchanged, if we adopt Bishop's technique, equation (3), instead of the original equation (1), remembering

(a) to decrease the pore pressures at all points below the external water level by the equilibrium head of water corresponding to the external water level as shown in Fig. 8; this line has no bearing on the phreatic line that actually exists in the soil

(b) to change the actual density to the submerged one for the soil below the line at the external water level

(c) to forget the external water completely.

This approach is merely convenient and does not change the equilibrium status of the mass of soil.

With the availability of rigorous methods such as that of Sarma (1973) which can be handled by longhand computations when necessary, it is no longer necessary to use simplified methods in practice. However, the Author's presentation is essential to explain to students and practitioners 


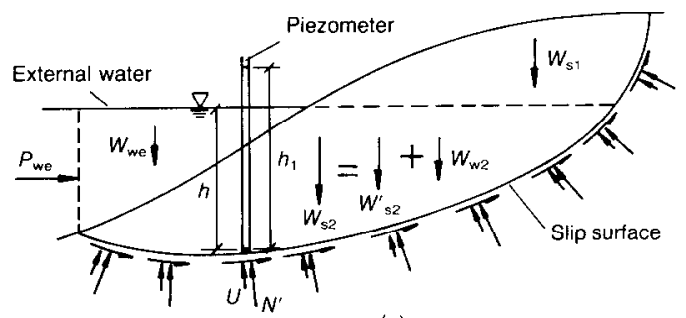

(a)

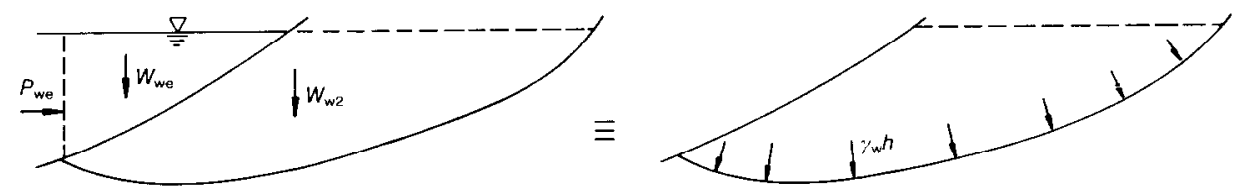

(b)

Fig. 8. (a) Overall situation and (b) external water and water load exert pressure on slip surface below external water level

the need to understand the mechanics of the solution of the method of slices and is therefore a welcome contribution.

\section{M. Morrison, L. G. Mouchel \& Partners}

\section{J. R. Greenwood, Travers Morgan}

In developing a revised method of slope stability analysis the Author has made two physical assumptions: that there are no effective inter-slice stresses, and a pore water assumption based on the seepage forces within the slices.

The seepage force on a two-phase mass of soil and water through which flow is occurring is given by the vector sum of the total weight of the soil's water mass, the resultant force contributed by all water pressures on the boundaries of the mass, and the resultant force caused by all effective pressures across the boundaries (Taylor, 1948). The Author has used this approach to argue that the water forces on a single slice apply a seepage force which must be resisted by effective pressures across the boundaries of the slice. This argument may be applied to the slide mass overall and, for equilibrium, the vector sum of the total weight of the slide mass, the effective and water pressures acting on the slip surface must be nil. Hence the seepage forces on the slices do not apply a disturbing force to the slide mass in addition to that already included by considering the total weight and the forces on the boundary of the slide mass. The significant effect of the seepage forces is to alter the distribution of the effective pressures along the slip surface.

In developing this revised method the Author has used the submerged weight and the seepage force acting on the slice to determine the normal and shear stresses on the slip surface. An alternative and more conventional approach is to consider the total weight and the boundary stresses (Taylor, 1948). In a rigorous analysis, both approaches will provide the same result, but this is not necessarily so in a simplified analysis. With the Author's simplifying assumption that there are no inter-slice effective stresses the two methods may be compared.

Resolving the total weight and boundary stresses normal to the slip surface, from Figs 2 and 3, the normal effective stress on the slip surface is

$$
N^{\prime}=W \cos \alpha+\Delta P_{w} \sin \alpha-u b / \cos \alpha
$$

and resolving parallel to the slip surface, the shear stress is

$$
T=W \sin \alpha+\Delta P_{w} \cos \alpha
$$

putting equation (17) equal to equation (26), substituting equation (19) and rearranging

$$
F_{\mathrm{s}}=\frac{\left(u b-b h \gamma_{\mathrm{w}} \cos ^{2} \alpha-\Delta P_{\mathrm{w}} \sin \alpha \cos \alpha\right)}{\cos \alpha \sin (\alpha-\beta)}
$$

Similarly, putting equation (18) equal to equation (27), substituting equation (19) and rearranging

$$
F_{\mathrm{s}}=\frac{\left(b h \gamma_{\mathrm{w}} \sin ^{2} \alpha-\Delta P_{\mathrm{w}} \sin \alpha \cos \alpha\right)}{\sin \alpha \cos (\alpha-\beta)}
$$

For an acceptable solution, resolving parallel and normal to the slip surface must give the same value of the seepage force $F_{\mathrm{s}}$. Equating equations 
(28) and (29), the following condition for an acceptable solution is obtained

$$
\begin{aligned}
\left(u b-b h \gamma_{w}\right. & \left.\cos ^{2} \alpha-\Delta P_{w} \sin \alpha \cos \alpha\right) \tan \alpha \\
= & \left(b h \gamma_{w} \sin ^{2} \alpha-\Delta P_{w} \sin \alpha \cos \alpha\right) \\
& \times \tan (\alpha-\beta)
\end{aligned}
$$

In general this condition is only satisfied when $u=h \gamma_{w}$ and $\beta=0$, i.e., under hydrostatic conditions when there is no seepage force.

In a real soil through which flow is occurring the seepage force is not zero and is resisted by effective stresses in the soil, and in particular along the inter-slice boundaries. Hence the assumption that there are no inter-slice effective stresses also implies that there are no seepage forces and that hydrostatic conditions exist within each slice. This is the assumption implied by the simple method (Greenwood, 1983).

In mathematically rigorous slope stability analysis, which includes the effective stresses on the inter-slice boundaries (Sarma, 1979), the pore water forces on the inter-slice boundaries are provided as part of the data specifying the stability problem. As a result, the effect on the factor of safety of the seepage forces within the slide mass may be studied directly. A similar approach for simplified analysis with no effective inter-slice stresses has been presented by Morrison \& Greenwood (1989). Separation of the seepage forces from the stability equations assists in understanding the slope stability problem. In practice the most common and simplest method of calculating the seepage force on a slice is from the pore water pressures on the boundaries of the slice.

\section{Author's reply}

The Author does not agree with Sarma that all rigorous methods are implicitly correct when inter-slice shear and normal forces are taken into account. This is because the water pressures and corresponding forces on the vertical sides of the slices are not computed correctly. The incremental water force across a slice depends on both the magnitude and direction of the seepage force as demonstrated by equations (7) and (14) and reiterated by Sarma (subject to a change of sign of $\Delta P_{w}$ ) in Fig. 7 . In the rigorous method proposed by Sarma (1973) it is assumed that, for all slices, the sum of the incremental total inter-slice forces is zero. This will not be so when seepage forces are present and this is essentially the same mistake which Taylor (1948) made with respect to inter-slice water forces.

Morrison and Greenwood are incorrect in stating that the Author has made two physical assumptions. Although inter-slice effective forces were neglected for the sake of clarity, the principles outlined in the Paper are not dependent on an assumption that they are zero. Further, no assumption was required to demonstrate the equivalence between lack of balance in the external water forces and the seepage forces which are carried by the soil by virtue of effective stresses. As stated above in reply to Sarma, the external water pressures on a slice are dependent on the magnitude and direction of the seepage force. Thus, although it is perfectly correct to use the total force polygon in any analysis, it is more convenient and direct to use the effective force polygon. Equation (30), derived by Morrison and Greenwood from the Author's Paper, is a correct identity although it does not appear to serve any useful purpose. It is of course satisfied when there is seepage as well as when groundwater is static. The Author cannot comprehend their following suggestion that an assumption that there are no inter-slice effective forces implies that there are no seepage forces.

The method of slices is inherently approximate and in the Author's opinion does not warrant excessive refinement. However, the theoretical forces due to seepage in slopes can be determined to any desired accuracy and should be included explicitly.

\section{REFERENCES}

Janbu, N. (1957). Earth pressures and bearing capacity calculations by generalized procedure of slices. Proc. 4th Int. Conf. Soil Mech. and Fndn Engng, 2, 207212.

Morgenstern, N. R. \& Price, V. E. (1965). The analysis of the stability of general slip surfaces. Géotechnique 15, No. 1, 79-93.

Morrison, I. M. \& Greenwood, J. R. (1989). Assumptions in simplified slope stability analysis by the method of slices. Géotechnique 39, No. 3, 503-509.

Sarma, S. K. (1973). Stability analysis of embankments and slopes. Géotechnique 23, No. 3, 423-433.

Sarma, S. K. (1979). Stability analysis of embankments and slopes. J. Geotech. Engng Div. Am. Soc. Civ. Engrs 105, GT12, 1511-1524.

Taylor, D. W. (1948). Fundamentals of soil mechanics. New York: Wiley/Chapman \& Hall. 